\title{
Use of FDG-PET Scan for the Assessment of Large Vessel Vasculitis
}

\section{Daniel Blockmans, MD, PhD}

\author{
Address \\ Department of General Internal Medicine, University Hospital Gasthuisberg, UZ \\ Gasthuisberg, Herestraat 49, B-3000, Leuven, Belgium \\ Email: daniel.blockmans@uzleuven.be
}

Published online: 11 April 2016

(C) Springer International Publishing AG 2016

This article is part of the Topical Collection on Vasculitis

Keywords Vasculitis - Large vessel vasculitis - Giant cell arteritis - Takayasu arteritis - Polymyalgia rheumatica . Temporal arteritis $\cdot$ Periaortitis · Fluorodeoxyglucose $\cdot$ Positron emission tomography $\cdot$ FDG $\cdot$ PET

Abbreviations AUC Area under the curve - CT Computerized tomography - FDG Fluorodeoxyglucose - GCA Giant cell arteritis - LVV Large vessel vasculitis - PET Positron emission tomography - PMR Polymyalgia rheumatic - SUV Standardized uptake value $\cdot R O C$ Receiver-operating characteristic . TBR Target to background ratio

\section{Opinion statement}

Temporal artery biopsy will only detect (part of) the cranial forms of giant cell arteritis. For the large vessel variant of this type of vasculitis, FDG-PET is the technique of choice. Since treatment of large vessel vasculitis implies long-term treatment with steroids, it is important to have a firm diagnosis. I suggest performing a FDG-PET scan in every patient in whom large vessel vasculitis is suspected and in whom the temporal artery biopsy is negative. In the follow-up of giant cell arteritis patients, we can rely on clinical symptoms and inflammatory parameters to monitor disease activity and to adapt our treatment and do not need to repeat FDG-PET scan. In Takayasu arteritis, follow-up FDG-PET scans are probably necessary to assess ongoing inflammation, but prospective studies are lacking.

\section{Introduction}

Seventeen years ago, the first report was published on the use of fluorodeoxyglucose (FDG)-positron emission tomography (PET) in the diagnosis of giant cell arteritis (GCA) and polymyalgia rheumatica (PMR) [1]. In the same year, other investigators reported on its use in that other large vessel vasculitis (LVV), Takayasu arteritis [2], while the group of Weyand and coworkers published a major article on the existence of a large vessel variant of GCA, in addition to its typical cranial form [3]. This large vessel variant of GCA can produce claudication of upper 
or lower limbs, in addition to very non-specific symptoms like weight loss, fever, and general malaise, making one consider GCA as a possible explanation for these complaints. FDG-PET scan was shown to be superior to gallium scanning in patients with GCA presenting with fever of unknown origin, due to its ability to demonstrate LVV [4]. After the year 2000, numerous case reports, and several retrospective and prospective studies have confirmed the potential of this isotopic technique in the diagnosis of LVV [5-10]. As a consequence of all these studies, temporal artery biopsy has lost its status as the gold standard for the diagnosis of GCA: one cannot expect that a procedure that samples only a few centimeters of a cranial artery could be diagnostic of a disease that may not involve the cranial arteries at all. FDG-PET can show inflammation of all major arteries throughout the body, but inflammation of the temporal arteries cannot be demonstrated on FDG-PET since these arteries are too small, too superficial, and too proximal to the FDG-consuming brain. Hence, temporal artery biopsy and FDG-PET scan can be regarded as complementary for the diagnosis of GCA. The role of FDG-PET in the follow-up of LVV is less clear. This poses a problem, especially for the management of Takayasu arteritis patients, who may develop arterial stenosis in the absence of clinical symptoms or inflammation parameters. In patients with chronic periaortitis, PET can also be used to show the extent of vasculitis [11]. Isolated PMR manifests itself on FDG-PET as an increase in FDG uptake around shoulders and hips, in combination with FDG accumulation in the cervical or lumbar spine; in about $30 \%$ of patients, slight vascular FDG uptake, especially in the subclavian arteries, can be seen as well [12].

This article will review major publications on the role of FDG-PET in the assessment of LVV from 2013 to 2015.

\section{Qualification or semiquantification of vascular and articular FDG uptake}

Most authors use a qualitative analysis of FDG uptake for assessing the presence of vasculitis, with the 18F-FDG uptake of the vessel wall being visually analyzed or compared to a reference structure, generally the liver, although occasionally the lung or a venous structure may be used. Semiquantitative methods relate arterial maximal standardized uptake value (SUVmax) values to a background activity (the so-called normalized arterial SUVmax) and define a cutoff value for the presence of vasculitis. Besson and colleagues found however that liver SUVmax values were significantly higher in GCA patients than in controls, which is a limitation to the use of the liver as a control and would favor the use of the lung as a reference structure [13•]. Cristina Puppo and colleagues recently published a systematic review, including 19 original articles representing a total of 442 GCA patients and 535 healthy controls. They described the different qualitative, semiquantitative, and combined methods that have been proposed for assessing the presence and grading the severity of GCA-related vascular inflammation. The majority of the included studies adopted qualitative methods of PET image analysis, which were found to be less sensitive but more specific than semiquantitative ones. Among the semiquantitative approaches, the aortic-to-blood pool uptake ratio of the aortic

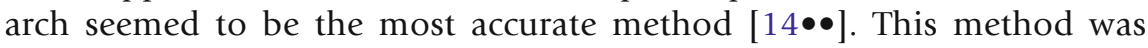
first developed by Rudd et al. [15] for the evaluation of arterial wall inflammation in atherosclerosis and then was used in 11 GCA patients and 11 controls by Besson et al., yielding a sensitivity of $81.8 \%$ and a specificity of $91 \%$ for vasculitis $[13 \bullet]$. 
Stellingwerff et al. compared two qualitative visual methods (first impression and vascular uptake versus liver uptake) and four semiquantitative methods (SUVmax aorta, SUVmax aorta-to-liver ratio, SUVmax aorta-tosuperior-caval-vein ratio, and SUVmax aorta-to-inferior-caval-vein ratio) for evaluation of FDG vascular uptake in 12 steroid-naive GCA patients, 6 GCA patients on steroid treatment, 18 inflammatory controls, 19 patients with atherosclerosis, and 16 normal controls. They concluded that a visual grading method with an arterial FDG uptake higher than the liver FDG uptake had the highest diagnostic accuracy for GCA. They also stressed the importance to score the pattern of FDG uptake (focal versus diffuse) and to correct for the presence of atherosclerosis. When using the semiquantitative methods, the best method was to calculate the aorta-to-liver SUVmax ratio. Furthermore, the use of steroids resulted in a less reliable aorta-to-liver SUVmax ratio [16].

In a study on 31 FDG-PET/CT scans, derived from 2 databases, Lensen et al. found the highest interobserver agreement (kappa is 0.96 in initial study and 0.79 in external validation) when diffuse vascular wall FDG uptake higher than liver uptake was used as a diagnostic criterion for LVV; this technique had both high sensitivity (100\%) and high specificity (98\%) [17].

Another point of discussion is the optimal time window between the injection of FDG and the acquisition of the images. Martinez-Rodriguez et al. reported that the images acquired after $180 \mathrm{~min}$ after injection provide a better contrast for the evaluation of the aortic wall than those obtained after $60 \mathrm{~min}$, due to the washout of the blood pool activity rather than increased FDG uptake

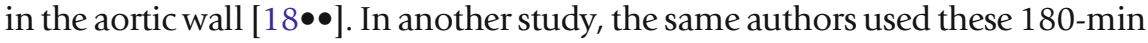
images to assess possible aortitis in 43 patients suspected of having LVV ( 25 of whom had a final diagnosis of aortitis) and in 15 oncological controls. A semiquantitative analysis was performed calculating the aortic wall SUVmax, the lumen SUVmax, and the target to background ratio. The mean aortic wall SUVmax was $2.00 \pm 0.62$ for patients with aortitis and $1.45 \pm 0.31$ for patients without aortitis $(p<0.0001)$. The target to background ratio (TBR) was 1.66 \pm 0.26 for patients with aortitis and $1.24 \pm 0.08$ for patients without aortitis $(p<0.0001)$. The differences were also statistically significant when the patients with aortitis and controls were compared. Receiver-operating characteristic (ROC) analysis revealed that the area under the curve (AUC) was greater for the TBR than for the aortic wall SUVmax (0.997 vs 0.871). The highest sensitivity and specificity were obtained for a TBR of 1.34 (sensitivity $100 \%$, specificity $94.4 \%)$ [19].

\section{Use of FDG-PET in the diagnosis and assessment of GCA and PMR}

Balink et al. performed FDG-PET/CT in a series of 140 patients with inflammation of unknown origin. Eighteen patients were diagnosed with PMR, and 12 were diagnosed with large vessel vasculitis. Signs of PMR were seen in three patients with LVV; contrariwise, signs of LVV were found on the PET images of four patients diagnosed with PMR. None of the 12 patients with LVV had classical clinical signs or symptoms of giant cell arteritis [20]. Another group of Dutch investigators reported on the use of FDG-PET in 88 patients older than 50 years with inflammation of unknown origin: They diagnosed 18 patients with LVV and 6 with PMR [21]. Both studies confirm our first report on the use 
of FDG-PET in fever of unknown origin [4], indicating once again that fever of unknown origin and inflammation of unknown origin are manifestations of the same diseases.

In a Japanese study, inflammation detected by PET at the ischial tuberosities, greater trochanters, and interspinous spaces discriminated between PMR and rheumatoid arthritis but not between PMR and spondyloarthritis, although the latter were more likely to have increased uptake in the sacroiliac joints. Hence, the topographic distribution and combination of high-uptake sites may differentiate these disorders [22]. In another study, uptake by the knee capsules was noted on PET-CT in 21 out of 25 patients with PMR. The uptake pattern seen in $96 \%$ of PMR patients was found in only $20 \%$ of controls $(P=0.03)$ [23•]. In an editorial published in Joint Bone Spine, Wendling et al. suggest that PET may be the ideal tool in PMR, in part due to its ability to reveal underlying malignancies [24].

Muto and colleagues from Tokyo, Japan, used a combination of FDG-PET/CT and contrast-enhanced CT for the early diagnosis of LVV in elderly patients with inflammation of unknown origin. In their retrospective analysis of $13 \mathrm{LVV}$ patients and 13 control patients, patients with LVV had significantly higher aortic wall SUVmax (3.85 versus 1.95) PET scores by FDG-PET/CT, and aortic wall thicknesses by contrast-enhanced CT (3.8 versus $2.6 \mathrm{~mm}$ ). During follow-up under treatment, PET scores and contrast-enhanced CT findings diminished [25].

The group of Maria Cid from Barcelona published the results of a prospective study using PET in 32 consecutive, biopsy-proven GCA patients. Twenty patients, who underwent PET/CT for cancer staging, served as controls. Mean SUVmax was significantly higher in patients than in controls in all vessels explored, and it correlated with acute-phase reactants and serum IL-6. The mean SUVmax of all vascular territories had an AUC of 0.830 and a cutoff of 1.89, yielding a sensitivity of $80 \%$ and a specificity of $79 \%$ for a diagnosis of GCA. FDG uptake in the supraaortic branches had the highest AUC. FDG uptake by the aorta showed a lower AUC, especially in the abdominal segment where atherosclerosis is more prevalent in the general population. [26•]. The fact that 17 out of 32 GCA patients had received high-dose steroids for up to 3 days may have obscured these results.

In a retrospective study on $17 \mathrm{LVV}$ patients (including 15 patients with GCA and 2 with Takayasu arteritis), Dellavedova and colleagues conclude that the intensity and the extension of increased FDG uptake in vessels at diagnosis (using 2 novel volume-based parameters: "volume of increased uptake" and "total lesion glycolysis") can predict the clinical course of the disease, separating patients with favorable or complicated progress [27]. This is in contrast to previous studies [8] and should be confirmed in a large prospective study.

\section{Use of FDG-PET in the diagnosis and assessment of Takayasu} arteritis

In a cross-sectional study, PET/CT scans were done in 22 patients with Takayasu arteritis and 17 of whom were treated with immunosuppression. In this study, only four patients had clinically active disease, and the PET scan confirmed the presence of active vasculitic lesions in all four patients. In 16 of the 18 patients who were in clinical remission, the PET/CT scans were normal. In two patients 
considered inactive, the PET scan showed increased vascular FDG uptake. Clinical exacerbation occurred 4 weeks later in one of these patients. Overall sensitivity and specificity of FDG PET/CT findings for clinical activity were 100 and $89 \%$, respectively [28].

A retrospective analysis from India was published of $60 \mathrm{PET} / \mathrm{CT}$ studies performed in 51 patients ( 17 scans were taken at the time of diagnosis, while 43 scans were taken after immunosuppression was initiated). PET/CT demonstrated active vasculitis in all 17 patients at the time of diagnosis, but only 14/43 scans $(32.5 \%)$ performed on patients receiving immunosuppression. There was a significant difference between the mean SUVmax and mean SUVratio at diagnosis and on immunosuppression, respectively $(p<0.01)$, whereas the median ESR levels did not differ. However, no firm conclusions can be drawn from this retrospective study: two-thirds of patients did not have an ESR available at the time of the PET scan, a large number of patients were not available for follow-up studies, and a positive PET scan itself was considered a proof of active disease, without taking into account clinical presentation [29].

\section{Use of FDG-PET in the diagnosis and assessment of other forms of} aortitis

In a retrospective study, Ebbo et al. evaluated FDG-PET/CT for disease staging and treatment evaluation in 21 patients with IgG4-related disease (three of whom also had aortitis) who underwent 46 PET/CT scans. They concluded that FDG-PET/CT results generally correlated with treatment response and disease activity [30].

Aortic inflammation was studied by PET/CT in 21 patients with GPA, 14 patients with sarcoidosis, 7 patients with head and neck carcinoma were used as healthy controls, and 5 patients with LVV. The mean TBRmax of the aorta was significantly higher in patients suffering from GPA (especially those with previous renal involvement) or LVV compared to healthy controls. The significance of this increased FDG uptake in patients suffering from small vessel vasculitis is not clear: is it pronounced atherosclerosis or large vessel vasculitis (i.e., arteritis of the vasa vasorum) [31]? In another journal, the same group reported aortic hot spots on FDG-PET in 8 out of 33 GPA patients [32].

\section{Review articles and meta-analyses on FDG-PET in vasculitis published in 2013-2015}

In an excellent review article, Balink et al. discuss the role of FDG-PET in the classification of LVV and they correctly state that several patients have been erroneously regarded in publications as Takayasu patients, despite an age over 50 , due to their aortic inflammation on FDG-PET $[33 \bullet \bullet]$. Another extensive review on the role of FDG-PET/CT in the diagnosis and management of systemic vasculitis (including medium sized vessel and small vessel vasculitis) was written by Danve and $\mathrm{O}^{\prime}$ Dell $[34 \bullet \bullet]$. 


\section{A new modality: fully integrated PET/MRI}

Yamashita et al. discuss the clinical value of PET/CT in several rheumatic diseases: not only in large vessel vasculitis including IgG4 disease and PMR but also in rheumatoid arthritis, spondyloarthritis, adult-onset Still's disease, relapsing polychondritis, granulomatosis

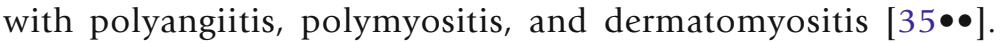

In January 2013, a meta-analysis was published that included six studies that used FDG-PET for the assessment of disease activity in Takayasu arteritis. The pooled sensitivity was $70 \%$, and the pooled specificity $77 \%$ [36].

Michael Soussan and colleagues from Paris recently published a metaanalysis on the value of FDG-PET that included 21 studies in a total of $413 \mathrm{LVV}$ patients and 299 controls. For GCA patients, a pooled sensitivity of $90 \%$ and a pooled specificity of $98 \%$ were calculated. The pooled sensitivity for Takayasu patients was $87 \%$ and the pooled specificity $73 \%$ ( $84 \%$ specificity with studies using National Institutes of Health criteria as the disease activity assessment scale). The authors mention that a moderate vascular FDG uptake in patients with Takayasu arteritis was observed in a significant number of patients with

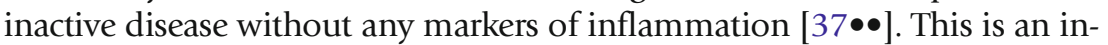
triguing observation, knowing that postmortem histological studies showed a significant amount of inflammation in patients considered to have inactive disease. Of course, this meta-analysis is not able to prove that this residual FDG uptake has any effect on future development of stenosis or dilatations.

Einspieler and colleagues reported the first use of a combined PET/MRI in ten patients with GCA and 2 patients with Takayasu arteritis. MRI was presumed to demonstrate vessel wall inflammation if at least two of these criteria were present: increased wall thickness, mural contrast enhancement, and wall edema. PET alone revealed abnormal FDG uptake in 86 vascular regions, MRI in combination with PET could identify 95 vasculitis regions. There were significant correlations between CRP and disease extent in PET alone and PET/MRI but not with contrast-enhanced magnetic resonance angiography. The authors conclude that hybrid PET/MRI is feasible in LVV and holds promise for precisely determining disease extent and disease activity, which may be especially interesting for following up Takayasu patients [38].

FDG PET/CT scan remains a valuable aid in the early diagnosis of LVV, when temporal artery biopsy is negative. There is still no consensus on which method should be used to report FDG findings: qualitative methods will continue to be used in clinical practice, while semiquantitative but time-consuming methods are more appropriate for clinical studies. Aortic wall SUVmax can be compared to the SUVmax of the liver or-probably better-to the blood pool SUVmax. This technique will not, however, detect those LVV vasculitis patients with isolated subclavian artery inflammation, in the absence of aortic involvement. Prospective studies on the use of FDG-PET in the follow-up of Takayasu arteritis are still lacking, and it is more difficult to say what role this technique should have in the longitudinal assessment of patients with this disease. 


\section{Compliance with Ethical Standards}

Conflict of Interest

DB declares that he has no conflicts of interest.

Human and Animal Rights and Informed Consent

This article does not contain any studies with human or animal subjects performed by the author.

\section{References and Recommended Reading}

Papers of particular interest, published recently, have been

highlighted as:

- Of importance

$\bullet \quad$ Of major importance

1. Blockmans D, Maes A, Stroobants S, Nuyts J, Bormans G Knockaert D, et al. New arguments for a vasculitic nature of polymyalgia rheumatica using positron emission tomography. Rheumatology (Oxford). 1999;38:444-7.

2. Hara M, Goodman PC, Leder RA. FDG-PET finding in early-phase Takayasu arteritis. J Comput Assist Tomogr. 1999;23:16-8.

3. Brack A, Matinez-Taboada V, Stanson A, Goronzy JJ, Weyand CM. Disease pattern in cranial and large-vesse giant cell arteritis. Arthritis Rheum. 1999;42:311-7.

4. Blockmans D, Knockaert D, Maes A, De Caestecker J, Stroobants S, Bobbaers H, et al. Clinical value of (18)Ffluoro-deoxyglucose positron emission tomography for patients with fever of unknown origin. Clin Infect Dis. 2001;32:191-6.

5. Blockmans D, Stroobants S, Maes A, Mortelmans L. Positron emission tomography in giant cell arteritis and polymyalgia rheumatic: evidence for inflammation of the aortic arch. Am J Med. 2000;108:1203-5.

6. Brodmann M, Lipp RW, Passath A, Seinost G, Pabst E, Pilger E. The role of 2-18F-fluoro-2-deoxy-D-glucose positron emission tomography in the diagnosis of giant cell arteritis of the temporal arteries. Rheumatology. 2003.

7. Moosig F, Czech N, Mehl C, Henze E, Zeuner RA, Kneba $\mathrm{M}$, et al. Correlation between 18-fluorodeoxyglucose accumulation in large vessels and serological markers of inflammation in polymyalgia rheumatica: a quantitative PET study. Ann Rheum Dis. 2004;63:870-3.

8. Blockmans D, de Ceuninck L, Vanderschueren S, Knockaert D, Mortelmans L, Bobbaers H. Repetitive 18F-fluorodeoxyglucose positron emission tomography in giant cell arteritis: a prospective study of 35 patients. Arthritis Rheum. 2006;55:131-7.

9. Lehmann P, Buchtala S, Achajew N, Haerle P, Ehrenstein B, Lighvani H, et al. 18F-FDG PET as a diagnostic procedure in large vessel vasculitis - a controlled, blinded re-examination of routine PET scans. Clin Rheumatol. 2011;30:37-42.
10. Hooisma GA, Balink H, Houtman PM, Slart RHJA Lensen KDF. Parameters related to a positive test result for FDG PET(/CT) for large vessel vasculitis: a multicenter retrospective study. Clin Rheumatol. 2012;31:861-71.

11. Salvarani C, Pipitone N, Versari A, Vaglio A, Serafini D, Bajocchi G, et al. Positron emission tomography (PET): evaluation of chronic periaortitis. Arthritis Rheum. 2005;53:298-303.

12. Blockmans D, De Ceuninck L, Vanderschueren S, Knockaert D, Mortelmans L, Bobbaers H. Repetitive 18fluorodeoxyglucose positron emission tomography in isolated polymyalgia rheumatica: a prospective study in 35 patients. Rheumatology (Oxford). 2007;46:672-7.

13.• Besson FL, de Boysson H, Parienti J, Bouvard G, Bienvenu B, Agostini D. Towards an optimal semiquantitative approach in giant cell arteritis: an 18FFDG PET/CT case-control study. Eur J Nucl Med Mol Imaging. 2014;41:155-66.

Interesting article in search for the optimal reporting of PET findings in LVV.

14.• Puppo C, Massollo M, Paparo F, Camellino D, Piccardo A, Naseri MSZ, et al. Giant cell arteritis: a systematic review of the qualitative and semiquantitative methods to assess vasculitis with $18 \mathrm{~F}$ fluorodeoxyglucose positron emission tomography. BioMed Res Int. 2014;2014:574248.

Very thorough article reviewing all major articles on this subject.

15. Rudd JHF, Myers KS, Bansilal S, et al. Atherosclerosis inflammation imaging with 18F-FDG PET: carotid, iliac, and femoral uptake reproducibility, quantification methods, and recommendations. J Nucl Med. 2008;49:871-8.

16. Stellingwerff MD, Brouwer E, Lensen KJDF, Rutgers A, Arends S, van der Geest KSM, et al. Different scoring methods of FDG PET/CT in giant cell arteritis Need for standardization. Medicine. 2015;94:e1542.

17. Lensen KDF, Comans EFI, Voskuyl AE, van der Laken CJ, Brouwer E, Zwijnenbrug AT, et al. Large-vessel vasculitis: interobserver agreement and diagnostic 
accuracy of 18F-FDG-PET/CT. BioMed Res Int. 2015;2015:914692.

18.• Martinez-Rodriguez I, del Castillo-Matos R, Quirce R, Jimenez-Bonilla J, De Arcocha-Torres M, Ortega-Nava F, et al. Comparison of early ( $60 \mathrm{~min}$ ) and delayed (180 min) acquisition of 18F-FDG PET/CT in large vessel vasculitis. Rev Esp Med Nucl Imagen Mol. 2013;32:222-6.

Important article pointing to a major until now unresolved question: the optimal time between tracer injection and acquisition of images.

19. Martinez-Rodriguez I, Martinez-Amador N, Banzo I, Quirce R, Jimenez-Bonilla J, de Arocha-Torres M, et al. Assessment of aortitis by semiquantitative analysis of 180-min 18F-FDG PET/CT acquisition images. Eur J Nucl Med Mol Imaging. 2014;41:2319-24.

20. Balink H, Bennink RJ, Veeger NJ, van Eck-Smit BL, Verberne HJ. Diagnostic utility of 18F-FDG PET/CT in inflammation of unknown origin. Clin Nucl Med. 2014;39:419-25.

21. Lensen KDF, Voskuyl AE, van der Laken CJ, Comans EFI, van Schaardenburg D, Arntzenius AB, et al. 18Ffluorodeoxuglucose positron emission tomography in elderly patients with an elevated erythrocyte sedimentation rate of unknown origin. PLoS ONE. 2013;8:e58917.

22. Yamashita H, Kubota K, Takahashi Y, et al. Similarities and differences in fluorodesoxyglucose positron emission tomography/computed tomography findings in spondylarthropathy, polymyalgia rheumatic and rheumatoid arthritis. Joint Bone Spine. 2013;80:171-7.

23. Cimmino MA, Camellino D, Paparo F, et al. High frequency of capsular knee involvement in polymyalgia rheumatica/giant cell arteritis patients studied by positron emission tomography. Rheumatology (Oxford). 2013;52:1865-72.

Interesting new finding in PMR, which had probably escaped our attention since the knees are normally not included in a regular PET scan.

24. Wendling D, Blagosklonov O, Boulahdour H, Prati C. Positron emission tomography: the ideal tool in polymyalgia rheumatica? Joint Bone Spine. 2014;81:381-3.

25. Muto G, Yamashita H, Takahashi Y, Miyata Y, Morooka $\mathrm{M}$, Minamimoto $\mathrm{R}$, et al. Large vessel vasculitis in elderly patients: early diagnosis and steroid-response evaluation with FDG-PET/CT and contrast-enhanced CT. Rheumatol Int. 2014;34:1545-54.

26. Prieto-Gonzalez S, Depetris M, Garcia-Martinez A, Espigol-Frigole G, Tavera-Bahillo I, Corbera-Bellata M, et al. Positron emission tomography assessment of large vessel inflammation in patients with newly diagnosed, biopsy-proven giant cell arteritis: a prospective, casecontrol study. Ann Rheum Dis. 2014;73:1388-92.

Large prospective study on the use of PET/CT in GCA. Unfortunately, many patients had been treated with rather high dose steroids for up to 3 days before the PET scan was performed. 27. Dellavedova L, Carletto M, Faggioli P, Sciascera A, Del Sole A, Mazzone A, et al. The prognostic value of baseline 18F-FDG PET/CT in steroid-naïve large-vessel vasculitis: introduction of volume-based parameters. Eur J Nucl Med Mol Imaging. 2015. doi:10.1007/ s00259-015-3148-9.

28. Karapolat I, Kalfa M, Keser G, Yalçin M, Inal V, Kumanlioglu K, et al. Comparison of F18-FDG PET/CT findings with current clinical disease status in patients with Takayasu's arteritis. Clin Exp Rheumatol. 2013;31 Suppl 75:S15-21.

29. Santhosh S, Mittal BR, Gayana S, Bhattacharya A, Sharma A, Jain S. F-18 FDG PET/CT in the evaluation of Takayasu arteritis: an experience from the tropics. J Nucl Cardiol. 2014;21:993-1000.

30. Ebbo M, Grados A, Guedj E, Gobert D, Colavolpe C, Zaidan $\mathrm{M}$, et al. Usefulness of 2-(18F)-fluror-2-deoxyD-glucose-positron emission tomography/computed tomography for staging and evaluation of treatment response in IgG4-related disease: a retrospective multicenter study. Arthritis Care Res. 2014;66:86-96.

31. Kemna MJ, Bucerius J, Drent M, Vöö S, Veenman M, van Paassen P, et al. Aortic 18F-FDG uptake in patients suffering from granulomatosis with polyangiitis. Eur J Nucl Med Mol Imaging. 2015;42:1423-9.

32. Kemna MJ, Vandergheynst F, Vöö S, Blocklet D, Nguyen T, Timmermans SAMEG, et al. Positron emission tomography scanning in anti-neutrophil cytoplasmic antibodies-associated vasculitis. Medicine 2015; 94. doi: 10.1097/MD747.

33.• Balink H, Bennink RJ, van Eck-Smit BLF, Verberne HJ. The role of 18F-FDG PET/CT in large vessel vasculitis: appropriateness of current classification criteria ? BioMed Res Int. 2014;2014:687608.

Excellent review paper on the subject.

$34 . \bullet$ Danve A, O'Dell J. The role of $18 \mathrm{~F}$ fluorodeoxyglucose positron emission tomography scanning in the diagnosis and management of systemic vasculitis. Int J Rheum Dis. 2015;18:714-24.

Excellent review paper on the subject.

35.• Yamashita H, Kubota K, Mimori A. Clinical value of whole-body PET/CT in patients with active rheumatic diseases. Arthritis Res Ther. 2014;16:423.

Excellent review paper on the subject.

36. Cheng Y, Lv N, Wang Z, Chen B, Dang A. 18-FDG-PET in assessing disease activity in Takayasu arteritis: a meta-analysis. Clin Exp Rheumatol. 2013;31 Suppl 75:S22-7.

37.• Soussan M, Nicolas P, Schramm C, Katsahian S, Pop G, Fain $\mathrm{O}$, et al. Management of large-vessel vasculitis with FDG-PET A systematic literature review and metaanalysis. Medicine. 2015;94:e622. doi:10.1097/MD. 0000000000000622.

Well-performed meta-analysis on the use of FDG-PET in GCA and in Takayasu arteritis.

38. Einspieler I, Thürmel K, Pyka T, Eiber M, Wolfram S, Moog P, et al. Imaging large vessel vasculitis with fully integrated PET/MRI: a pilot study. Eur J Nucl Med Mol Imaging. 2015;42:1012-24. 